\title{
173. On a Conjecture of K. S. Williams
}

\author{
By Saburô UchiYama \\ Department of Mathematics, Shinshû University, Matsumoto \\ (Comm. by Kinjirô KUnUGi, M. J. A., Sept. 12, 1970)
}

1. Let $p$ be a rational prime and $n$ a positive integer $\geqq 2$. We denote by $a_{n}(p)$ the least positive integral value of $a$ which makes the polynomial $x^{n}+x+a$ irreducible $(\bmod p)$. In a recent paper [3] K. S. Williams conjectured that for all $n \geqq 2$ one has

$$
\liminf _{p \rightarrow \infty} a_{n}(p)=1,
$$

and showed (among others) that (1) is true for $n=2$ and 3 . In the present note we shall prove that (1) is true for $n=4,6,9,10$ and for all primes $n \equiv 1(\bmod 3)$. However, it is immediately clear that (1) is not true for some (in fact, infinitely many) values of $n$. Indeed, the polynomial $x^{n}+x+1$ is irreducible in $Z[x]^{*)}$ if and only if $n=2$ or $n \not \equiv 2$ $(\bmod 3)$, and for $n \equiv 2(\bmod 3) x^{n}+x+1$ has the obvious factor $x^{2}+x+1$ (cf. [2]). Thus, we can show that for $n=5$

$$
\liminf _{p \rightarrow \infty} a_{5}(p)=3
$$

and for $n=8$

$$
\liminf _{p \rightarrow \infty} a_{8}(p)=2 .
$$

2. Our foundation is on the following important theorem due to F. G. Frobenius [1].

Theorem. Let $f(x)$ be a square-free polynomial (i.e. a polynomial with non-zero discriminant) of degree $n \geqq 1$ in $Z[x]$, and let $d_{1}, \cdots, d_{r}$ $(r \geqq 1)$ be positive integers with $d_{1}+\cdots+d_{r}=n$. Then, if the Galois group of $f(x)$, as a permutation group on n letters, contains a permutation which is decomposed as the product of $r$ cycles of length $d_{1}, \cdots, d_{r}$, there are infinitely many primes $p$ such that we have

$$
f(x) \equiv f_{1}(x) \cdots f_{r}(x) \quad(\bmod p)
$$

where $f_{1}(x), \cdots, f_{r}(x)$ are polynomials of $Z[x]$, each irreducible $(\bmod p)$, of degree $d_{1}, \cdots, d_{r}$, respectively.

In fact, it is proved in [1] that the Dirichlet density of prime numbers $p$ for which (4) holds equals the number of permutations in the Galois group of $f(x)$ that have $r$ cycles of length $d_{1}, \cdots, d_{r}$, divided by the order of the group.

By virtue of this theorem, a simple and well-known argument on the reduction $(\bmod p)$ of the Galois group of $f(x)$ will show that the

*) We denote by $Z$, as usual, the ring of rational integers. 
existence of infinitely many primes $p$ satisfying (4) is equivalent to the existence of at least one such prime $p$ (not dividing the discriminant of $f(x)$ ).

The following result is a particular case of the above theorem.

Corollary. Let $f(x)$ be a polynomial of degree $n \geqq 1$ in $x$ with coefficients in $Z$. If the Galois group of $f(x)$ contains a cycle of length equal to $n$, then there are infinitely many primes $p$ for which $f(x)$ is irreducible $(\bmod p)($ so that $f(x)$ is necessarily irreducible in $Z[x])$.

Another interesting consequence of the theorem of Frobenius is that if $f(x)$ is an irreducible polynomial of degree $n \geqq 2$ in $Z[x]$, then there exists an infinity of primes $p$ such that the congruence

$$
f(x) \equiv 0 \quad(\bmod p)
$$

has no solution $x$ in $Z$. On the other hand, it is not difficult to see that for an arbitrary non-constant polynomial $f(x)$ in $Z[x]$ there are infinitely many primes $p$ for which the congruence (5) has solutions $x$ in $Z$.

3. Now, we shall apply the corollary to the theorem of Fobenius, to the special polynomials $x^{n}+x+a, a \in Z$. The polynomial $x^{n}+x+1$ is irreducible in $Z[x]$ for $n \equiv 1(\bmod 3)$. Hence, if $n \equiv 1(\bmod 3)$ is prime, then there are infinitely many primes $p$ for which $x^{n}+x+1$ is irreducible $(\bmod p)$. In order to obtain the other results enunciated in $\S 1$, it will suffice to find the least positive value of $a$ and an appropriate prime number $p$ such that $x^{n}+x+a$ is irreducible $(\bmod p)$. Thus, the polynomial $x^{n}+x+1$ is irreducible $(\bmod 2)$ for $n=2,3,4,6$, 9 and 10. Hence, (1) holds true for these values of $n$. Next, we see that the polynomial $x^{5}+x+3$ is irreducible $(\bmod 7)$, so that (2) holds. Finally, the polynomial $x^{8}+x+2$ is irreducible $(\bmod 3)$ and (3) holds.

Also, we can argue for the case of $n=6$ in the following way. We find that

and

$$
x^{6}+x+1 \equiv(x+2)\left(x^{2}+2 x+2\right)\left(x^{3}+2 x^{2}+x+1\right) \quad(\bmod 3)
$$

$$
x^{6}+x+1 \equiv(x+2)\left(x^{5}+5 x^{4}+4 x^{3}+6 x^{2}+2 x+4\right) \quad(\bmod 7),
$$

the factors on the right-hand side being irreducible to the respective moduli. It follows from this that the Galois group of $x^{6}+x+1$ is the symmetric group of degree 6 . (Here, use was made of the elementary fact that, if a transitive permutation group on $n$ letters contains a transposition and a cycle of length $n-1$, then the group coincides with the symmetric group of degree $n$.) Thus, the density of prime numbers $p$ for which $x^{6}+x+1$ is irreducible $(\bmod p)$ is equal to $1 / 6$.

Our method could of course be extended to treat the case of $n>10$, unless we avoided the incleasing complication with $n$ in the reduction of the relevant polynomials with various moduli (except for primes $n \equiv 1(\bmod 3))$. 
Added in proof (September 21, 1970). It is not difficult to see that the polynomial $x^{n}+x+a, a \in Z$; is irreducible in $Z[x]$ for $a=3$ and $n \geqq 2$ (for $a=2$ and even $n \geqq 2$ ). We thus have, in particular,

$$
\liminf _{p \rightarrow \infty} a_{n}(p)=3
$$

for every odd prime $n \equiv 2(\bmod 3)$.

\section{References}

[1] F. G. Frobenius: Über Beziehungen zwischen den Primidealen eines algebraischen Körpers und den Substitutionen seiner Gruppe. Sitzungsberichte Kön. Preuss. Akad. Wiss. Berlin, 689-703 (1896).

[2] E. S. Selmer: On the irreducibility of certain trinomials. Math. Scand., 4, 287-302 (1956).

[3] K. S. Williams: On two conjectures of Chowla. Canad. Math. Bull., 12, 545-565 (1969). 